Analyse: Ber. für $\mathrm{C}_{9} \mathrm{H}_{6} \mathrm{~N}_{3} \mathrm{O}_{2}$.

$$
\begin{aligned}
& \text { Procente: } \mathrm{Ag} 36.5 . \\
& \text { Gef. } \gg 36.3 .
\end{aligned}
$$

(1)-Phenylpyrazolon-(4)-Azobenzol,

$$
\mathrm{C}_{6} \mathrm{H}_{5} \cdot \mathrm{N}_{\mathrm{CO}}^{\mathrm{N}: \mathrm{N} . \mathrm{NHC} \mathrm{H}_{5}}=\mathrm{C}_{15} \mathrm{H}_{12} \mathrm{~N}_{17} \mathrm{O} \text {. }
$$

Nach üblicber Metbode erbältlich. Scbmp. 149-150. Gelbbraune körnige Krystalle oder Blättchen. Bereits von Knorr ${ }^{1}$ ) aus dem Osazon der Phenylbydrazidooxybrenztraubensäure erhalten.

Analyse: Ber. für $\mathrm{C}_{15} \mathrm{H}_{12} \mathrm{~N}_{4} \mathrm{O}$.

$$
\begin{aligned}
& \text { Procente: N } 21.2 . \\
& \text { Gef. } \gg 21.5 .
\end{aligned}
$$

Das Studium der nicht (1)-phenylirten Pyrazolidone gedenke ich fortzusetzen, um weitere Vergleiche mit entsprechenden Pyrazolonderivaten ziehen zu können.

550. J. T. Hewitt: Isomerie in der Azoreihe.

\title{
[Vorlänfige Mittheilung.]
}

(Eingegangen am 6. December; mitgetheilt in der Sitzung von Hrn. W. Will.)

Vor einiger Zeit habe ich das Orthochlorphenylhydrazin dargestellt und einige seiner Derivate im Journal of the Chemical Society beschrieben ${ }^{2}$ ). Es war zu erwarten, dass bei der Einwirkung dieses Hydrazins auf Chinone dieselben Körper entstehen würden, wie bei der Einwirkung des diazotirten Orthochloranilins auf die entsprechenden Phenole.

Die bei Anwendung des gewöhnlichen Phenols nach letzterer Reaction entstehende Azoverbindung zeigte ein Verbalten, welches zu näherem Studium einlud. Behufs Darstellung des Orthochlorbenzolazophenols wurden $30 \mathrm{~g}$ Orthochloranilinnitrat, gelöst in 21/2 L Wasser, mit $24 \mathrm{~g}$ Phenol versetzt und zu der Mischung eine Lösung ron $40 \mathrm{~g}$ Kaliumnitrit in $4 \mathrm{~L}$ Wasser hinzagefügt. Nach 20 Stunden wurde der harzige Niederschlag abfiltrirt, mit verdünntem Ammoniak ausgekocht und die filtrirte ammoniakalische Lösung angesäuert. Beim Erkalten schieden sich hellgelbe Nadeln aus, die behufs Reinigung in Alkohol gelöst wurden; wenig Wasser fälte

1) L. Knorr, Diese Berichte 21, 1204.

2) Journal of the Chemical Society 59,$209 ; 63,868$. 
zunächst etwas Harz, und auf weiteren Zusatz von Wasser erfüllte sich die Lösung mit feinen gelben Nadeln, welcbe constant bei $85^{\circ}$ schmolzen. Die Analyse lieferte auf die Formel des Orthochlorbenzol(12) phenols, $\mathrm{C}_{12} \mathrm{H}_{9} \mathrm{~N}_{2} \mathrm{OCl}$, stimmende Zahlen.

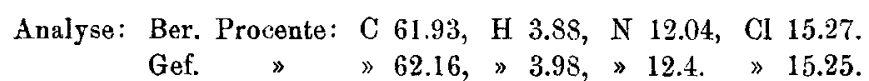

Ortbochlorbenzolazophenol ist in den gewöhnlichen orgaaischen Lösungsmitteln sehr leicht, in Wasser kaum löslich, aus der alkalischen Lösung wird es durch Kohlendioxyd niedergeschlagen. Wird die Verbindung eine Stunde lang auf $80^{\circ}$ erhitzt, so erleidet sie eine interessante Umwandlung, die gelbe Farbe macht einer hellrothen Platz und der Körper schmilzt nunmehr bei $96^{\circ}$. Folgende analytische Daten zeigen, dass die Zusammensetzong nicht geändert ist.

Analyse: Ber. Procente: C 61.93, H 3.88, N 12.04, Cl 15.27. Gef. " 》62.07, "3.81, 》12.85, 》 15.53 .

Die rothe Modification löst sich in denselben Lösungsmitteln wie die gelbe, aus ibrer Lösung in Alkali wird jedoch durch Säuren und gleicbfalls ans alkoholisehen durch Zusatz von Wasser die gelbe Modification abgeschieden.

Diese Erscheinung lässt sich so deuten, dass man den beiden Modificationen folgende tautomere Formeln zuschreibt:
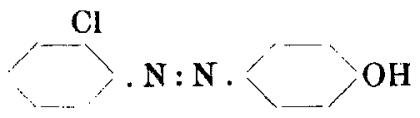

und

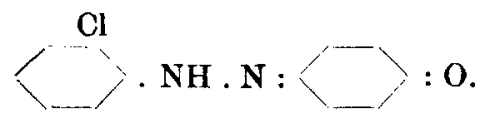

Ein solcher Tautomerismus sollte auch bei dem Benzolazo- $\alpha-$ naphtol auftreten, welches sowobl durch Einwirkang von Phenylhydrazin auf $\alpha$-Naphtochinon ${ }^{1}$ ), als auch durch Versetzen von salzsaurem Diazobenzol mit $\alpha-\mathrm{Naphtol}^{2}$ ) entsteht. Gleichwohl liegt nach $Z$ incke's Angabe Identität vor.

Es wäre jedoch noch eine andere Deutung möglich, die Isomerie dieser zwei Verbindungen liesse sich mit der Hantzsch'schen Hypothese in Einklang bringen und köninte durch folgende Formeln ausgedrückt werden:
$\mathrm{C}_{6} \mathrm{H}_{4} \mathrm{Cl} \cdot \stackrel{\mathrm{N}}{\dddot{N}}$
$\mathrm{C}_{6} \mathrm{H}_{4} \mathrm{OH} . \ddot{\mathrm{N}}$
und
$\mathrm{C}_{6} \mathrm{H}_{4} \mathrm{Cl} \cdot \mathrm{N}$
$\ddot{N} . \mathrm{C}_{6} \mathrm{H}_{4} \mathrm{OH}$.

In beiden Fällen liessen sich zwei isomere Acetylverbindungen erwarten, und man konnte durch das Studium der Reductionsproducte jener Acetylderivate die Frage bezüglich der Constitution der beiden Modificationen entscheiden. Es ist mir jedoch nicht gelungen, zwei verschiedene Acetylderivate darzustellen.

1) Zincke und Bindewald, diese Berichte 17, 3026.

) Typke, dicse Berichte 10, 1580. 
Orthochlorbenzolazophenylacetat entsteht aus beiden Modificationen durch Erhitzen mit überschüssigem Essigsäureanbydrid; aus verdünntem Weingeist umkrystallisirt, wird es in feinen gelben Nadeln erhalten, welche bei $100^{0}$ schmelzen.

Analyse: Ber. Procente: N 10.2.

Gef. $\gg \gg 10.4$.

Die Substanz ist unlöslich in Wasser, wird jedoch von Alkohol, Aether, Methylalkohol, Schwefelkohlenstoff und Benzol aufgenommen. Ammoniak wirkt bei gewöhnlicher Temperatur nicht auf die Acetylverbindung, beim Erbitzen hingegen tritt leicht Hydrolysirung ein.

Das Orthochlorbenzolazophenylbenzoat lässt sich nach der Schotten-Baumann' schen Methode berstellen und krystallisirt aus heissem Alkohol in gelben Blättchen, die bei $131^{\circ}$ schmelzen.

Analyse: Ber. Procente: N 8.32.

Diese Verbindung löst sich in Aether, Schwefelkohlenstoff, Benzol und Holzgeist, aber nicht in Wasser.

Das Verhalten des Orthochlorbenzolazophenols veranlasste mich, auch die entsprechende Meta- und Paraverbindung zu untersuchen. Das Metachlorbenzolazophenol fält aus der alkalischen Lösung beim Ansäuern in bläulich - rothen Flocken und krystallisirt aus verdünntem Alkohol in prachtvollen braun-violetten Nadeln.

Analyse: Ber. Procente: N 12.04.

Beim Erhitzen derselben tritt gleichfalls Farbenwechsel ein, der hier noch schärfer ist wie bei dem entsprechenden Orthokörper. Gegen $80^{\circ}$ wird die Substanz hellgelb, sie schmilzt dann bei $135^{\circ}$.

Analyse: Ber. Procente: N 12.04.

Gef. $》$ "12.7.

Das Azophenol löst sich leicht mit hellgelber Farbe in Alkohol, Benzol, Aceton, Eisessig, Aether, Chloroform und $\mathrm{X}_{y l o l}$, nicht so leicht in Schwefelkoblenstoff; von Alkalien wird es mit dunkler Farbe aufgenommen, ans dieser Lösung wird der Körper durch Säuren in rothen Nadeln gefällt. Das Absorptionsspectrum dieser Lösungen werde ich später untersucben.

Metachlorbenzolazophenylacetat entsteht durch Erhitzen des Phenols mit Natriumacetat und Essigsäureanbydrid. Es scheidet sich aus verdünntem Alkohol in schönen gelb-rothen Blättchen ab, die bei $92^{\circ}$ schmelzen.

Analyse: Ber. Procente: N 10.20.

Gef. $\gg \gg 10.26$.

Metachlorbenzolazophenylbenzoat wird nach dem Schotten-Baumann'schen Verfahren aus der gelben Modification erhalten und krystallisirt aus verdünntem Alkohol in gelben Schüppchen, die bei $118^{\circ}$ schmelzen. 
Analjse: Ber. Procente: N 8.32.
Gef. $》$ 》 8.54.

Parachlorbenzolaz ophenol wurde schon vor einigen Jabren aus Parachlordiazoamidobenzol und Phenol von Henmann und Oeconomides dargestellt. Ich habe es durch Versetzen von diazotirtem salzsanrem Parachloranilin mit Phenol erhalten und den Schmelzpunkt bei $154^{\circ}$ beobachtet, während die genannten Chemiker denselben bei $151-152^{\circ}$ angeben. Eine zweite Modification dieser Substanz entsteht nicht beim Erhitzen. Zur weiteren Charakterisirung dieses Körpers habe ich das Acetat und das Benzoat dargestellt.

Das Acetat scheidet sich aus Alkohol in haarfeinen gelben Nadeln ans, die bei $160^{\circ}$ schmelzen und in Alkohol nicht so löslich sind wie die entsprechende Metaverbindung.

$$
\begin{gathered}
\text { Analyse: Ber. Procente: N } 10.2 . \\
\text { Gef. } \gg \gg 10.4 .
\end{gathered}
$$

Das Benzoat, nach dem Schotten-Baumann'schen Verfahren dargestellt, bildet kleine gelhrothe Blättchen, die bei $154^{\circ}$ schmelzen. In kaltem Alkohol ist die Substanz nur wenig, in heissem jedoch ziemlich leicht löslich.

Analyse: Ber. Procente: N 8.32.

Gef. $\gg \gg 8.68$.

Ich gedenke diese interessanten Azo-Isomeren weiter zu untersuchen und hoffe, die Resultate dieses Studiums im Laufe dieses Winters im Journal of the Chemical Society zu veröffentlichen.

Cambridge. University Chemical Laboratory.

560. Eug. Bamberger und F. Kuhlemann:

Ueber das Diformazyl und seine Beziehung zum Diamidrazon. (Eingegangen am 7. December; mitgetheilt in der Sitzung von Hrn. W. Will.) [XI. Mittheilung über gemisehte Azokörper.]

Als die Gesetzmässigkeiten zusammengefasst wurden $^{1}$ ), welche die Wechselwirkung zwischen Aldehyden und Ketonen einerseits und Diazoverbindungen andererseits beherrschen, wurde unter anderem bemerkt, dass Ketoverbindungen mit den Atomgruppen ( $\mathrm{CO} . \mathrm{CH}_{3}$ ) resp. $\left(\mathrm{CO}, \mathrm{CH}_{2}, \mathrm{CO}\right)$ bei erschöpfender Behandlung mit alkalischem $\mathrm{N}_{2} \mathrm{C}_{6} \mathrm{H}_{5}$

Diazobenzol Phenylazoformazyl, $\mathrm{C}-\mathrm{N}_{2} \mathrm{H}_{6} \mathrm{H}_{5}$, Ketone und Aldehyde $\mathrm{N}_{2} \mathrm{C}_{6} \mathrm{H}_{5}$ dagegen, welche den Complex $\left(\mathrm{CO} . \mathrm{CH}_{2} \cdot \mathrm{CH}_{2} \cdot \mathrm{CO}\right)$ entbalten,

1) Diese Berichte 25, 3207. Ueber die Abspaltbarkeit aromatischer Alkyle S. 3210 und diese Berichte 25, 